\title{
State of the art: refinement of multiple sequence alignments
}

\author{
Saikat Chakrabarti , Christopher J Lanczycki, Anna R Panchenko, Teresa M Przytycka, Paul A Thiessen, \\ Stephen H Bryant
}

\begin{abstract}
Correction to Chakrabarti S, Lanczycki CJ, Panchenko AR, Przytycka TM, Thiessen PA and Bryant SH: State of the art: refinement of multiple sequence alignments. BMC Bioinformatics 2006, 7:499.
\end{abstract}

\section{Correction}

After publication of [1] we have noticed an error in our manuscript. We have realized that there were some numbers incorrectly inserted within the Table 1 of the manuscript [1]. Corrected values are now provided In
Table 1, for six of the numbers that were entered incorrectly: the SP scores for References 1, 4 and 5 under Muscle reference alignment (REFINER refinement alignment) and, SP scores for References 2, 3 and 5 under $\mathrm{T}$-Coffee reference alignment (Default refinement

Table 1 Impact on alignment quality following refinement.

\begin{tabular}{|c|c|c|c|c|c|c|c|c|c|c|c|c|}
\hline \multirow{2}{*}{$\begin{array}{l}\text { BAliBASE } \\
\text { reference } \\
\text { alignments }\end{array}$} & \multicolumn{4}{|c|}{ ClustalW } & \multicolumn{4}{|c|}{ Dialign } & \multicolumn{4}{|c|}{ Mafft } \\
\hline & Default & RASCAL & $\mathrm{RF}$ & REFINER & Default & RASCAL & $\mathrm{RF}$ & REFINER & Default & RASCAL & $\mathrm{RF}$ & REFINER \\
\hline Reference 1 & 0.65 & 0.63 & 0.66 & 0.66 & 0.62 & 0.65 & 0.67 & 0.62 & 0.70 & 0.69 & 0.69 & 0.71 \\
\hline Reference 2 & 0.78 & 0.80 & 0.80 & 0.80 & 0.78 & 0.80 & 0.79 & 0.79 & 0.83 & 0.82 & 0.83 & 0.82 \\
\hline Reference 3 & 0.66 & 0.69 & 0.67 & 0.68 & 0.65 & 0.64 & 0.65 & 0.66 & 0.76 & 0.73 & 0.75 & 0.77 \\
\hline Reference 4 & 0.67 & 0.68 & 0.66 & 0.70 & 0.67 & 0.71 & 0.66 & 0.69 & 0.75 & 0.73 & 0.70 & 0.77 \\
\hline Reference 5 & 0.65 & 0.67 & 0.66 & 0.68 & 0.67 & 0.65 & 0.64 & 0.67 & 0.76 & 0.73 & 0.72 & 0.76 \\
\hline Average & 0.682 & 0.694 & 0.69 & 0.704 & 0.678 & 0.690 & 0.682 & 0.692 & 0.760 & 0.740 & 0.738 & 0.766 \\
\hline $\begin{array}{l}\text { Increment } \\
(\%)\end{array}$ & 0 & 1.760 & 1.173 & 3.226 & 0.000 & 1.770 & 0.590 & 2.065 & 0.000 & -2.632 & -2.895 & 0.789 \\
\hline \multirow{2}{*}{$\begin{array}{l}\text { BAliBASE } \\
\text { reference } \\
\text { alignments }\end{array}$} & \multicolumn{4}{|c|}{ Muscle } & \multicolumn{4}{|c|}{ Probcons } & \multicolumn{4}{|c|}{ T-Coffee } \\
\hline & Default & RASCAL & $\mathrm{RF}$ & REFINER & Default & RASCAL & $\mathrm{RF}$ & REFINER & Default & RASCAL & $\mathrm{RF}$ & REFINER \\
\hline Reference 1 & 0.66 & 0.66 & 0.67 & 0.68 & 0.72 & 0.72 & 0.70 & 0.73 & 0.68 & 0.68 & 0.69 & 0.68 \\
\hline Reference 2 & 0.80 & 0.81 & 0.80 & 0.80 & 0.83 & 0.83 & 0.82 & 0.82 & 0.8 & 0.83 & 0.82 & 0.82 \\
\hline Reference 3 & 0.71 & 0.71 & 0.71 & 0.73 & 0.76 & 0.73 & 0.75 & 0.77 & 0.61 & 0.62 & 0.62 & 0.64 \\
\hline Reference 4 & 0.71 & 0.72 & 0.68 & 0.74 & 0.77 & 0.75 & 0.71 & 0.77 & 0.71 & 0.71 & 0.70 & 0.72 \\
\hline Reference 5 & 0.70 & 0.71 & 0.67 & 0.72 & 0.76 & 0.74 & 0.71 & 0.75 & 0.72 & 0.73 & 0.69 & 0.74 \\
\hline Average & 0.716 & 0.722 & 0.706 & 0.734 & 0.768 & 0.754 & 0.738 & 0.77 & 0.704 & 0.714 & 0.704 & 0.720 \\
\hline $\begin{array}{l}\text { Increment } \\
(\%)\end{array}$ & 0.000 & 0.838 & -1.397 & 2.514 & 0.000 & -1.823 & -3.906 & 0.260 & 0.000 & 1.420 & 0.000 & 2.273 \\
\hline
\end{tabular}

\footnotetext{
* Correspondence: chakraba@mail.nih.gov

National Center for Biotechnology Information, National Library of Medicine,

National Institutes of Health, Bethesda, MD 20894, USA
} 
alignment). However, this does not affect our original interpretation of the data presented in our original publication in any way. We regret any inconvenience that this inaccuracy might have caused.

Received: 10 November 2009

Accepted: 4 January 2010 Published: 4 January 2010

\section{References}

1. Chakrabarti S, Lanczycki CJ, Panchenko AR, Przytycka TM, Thiessen PA,

Bryant SH: State of the art: refinement of multiple sequence alignments. BMC Bioinformatics 2006, 7:499.

doi:10.1186/1471-2105-11-3

Cite this article as: Chakrabarti et al:: State of the art: refinement of multiple sequence alignments. BMC Bioinformatics 2010 11:3.

Submit your next manuscript to BioMed Central and take full advantage of:

- Convenient online submission

- Thorough peer review

- No space constraints or color figure charges

- Immediate publication on acceptance

- Inclusion in PubMed, CAS, Scopus and Google Scholar

- Research which is freely available for redistribution

Submit your manuscript at www.biomedcentral.com/submit
C BioMed Central 